\title{
Novel Approach to Estimate the SV Traffic Streaming Latency Based on IEC 61850-9-2LE
}

\author{
Mike Mekkanen $^{1}$, Erkki Antila ${ }^{2}$, Reino Virrankoski ${ }^{1}$ and Mohammed Elmusrati ${ }^{1}$ \\ ${ }^{1}$ University of Vaasa, Department of Computer Science \\ ${ }^{2}$ Department of Electrical Engineering
}

\begin{abstract}
Substation Automation System (SAS) performance has been gained great improvements by implementing the high data rate process bus based on IEC 61850-9-2LE. These improvements can be summarized within the most important such as reduction in the SAS constriction cost by eliminating the high cost copper field wiring, as one pair of optic fiber may handle all the communication, control and protection signals for a single intelligent electronic device (IED) that may replace 100 or more copper wire connections. Improving system performance and safety by keeping the high analog (voltages and current) signals within the SAS primary side, while provide a full digital SAS within the secondary side (bay and station levels). This paper illustrates and discusses the novel approach to estimate the sample value (SV) packets stream latency within the process bus network. Moreover, it shows the successful implementation of the novel SV packets stream latency estimation approach based on designing a practical IEC 61850-9-2LE process bus using commercial physical devices to assess their performance operation within the SAS.
\end{abstract}

KEYWORD: IEC61850-9-2LE; process bus; Merging Unit (MU); substation Automation system (SAS); Sample Value

\section{INTRODUCTION}

The wide internationally acceptance of the IEC61850 standard and the growth of IEC61850-9-2 process bus as a high data rate communication network have a great improved the capability of the SAS. These improvements can be summarized in two simple way as follows. Reduction in the SAS constriction cost by eliminating the high cost copper field wiring, as one pair of optic fiber may handle all the communication, control and protection signals for single intelligent electronic device (IED) that may replace 100 or more copper wire connections. Moreover, Improving system performance and safety by keeping the high analog (voltages and current) signals within the SAS primary side, while provide a full digital SAS within the secondary side (bay and station levels). IEC61850-9-2 provides distributed measurements for monitoring, control and protection functions by using so-called Merging Unit (MU). The MU has the ability to connect the analog word to the digital word within the SAS by converting the analog data signals that gathered from the primary instruments currents and voltages transformers $(\mathrm{CT}, \mathrm{VT})$ into digitalized standard packets for subsequent transmission through the process bus local area network (LAN). It can transmit over the point-to-point type connection to any IED or broadcast over the LAN in the similar manner as General Object Oriented Substation Events (GOOSE) messages. According to the IEC61850-9-2 that specified how SV measurements can be transmitted over the LAN whereas IEC61850-9-2LE (Light Edition) reduces the complexity and difficulty of implementation associated within the first version [1] [2]. This improvement has been achieved by restricting the data sets that are transmitted and specifying the sampling rates. The assigned dataset comprises four voltages and currents three phases and neutral for each as illustrated in Fig 1. Process bus network has been growing rapidly based on product development and commission process. However, regardless to this growth the knowledge about the real behavior of the process bus network, especially when there is a large number of traffic resources are connected within the same network is few in comparison with rapid growth of the process bus. The process bus network analysis is in the focus of research area from both industrial and academia, several network process bus modeling have been under testing, however their hard assumption limit their effectiveness [3]. In [4] modeling and simulation of the distribution substation $69 \mathrm{kV}$ and $220 \mathrm{kV}$, however the raw data of the SV traffic 
characteristic that been used is uncompliant with IEC61850-9-2LE. Where as in [5] several works have been done based on test bench that calculate and compare the characteristic of the SV traffic differences between the two baths direct from merging unit and the source of the digital reference signal. David Ingram has been done several works that discussed the proses bus critical issues such as time synchronization, routing and process bus traffic analysis. Most of the work had been done by using the GTNET card with the SV firmware to generate the MU stream and the Endace DAG7.5G4 network card to monitor the traffic. The weak point of these works they cannot reflect the real behavior of the system since they generate the streaming traffic based on mathematical calculation (number of $\mathrm{MU}$ within the network multiply by the traffic that each MU can generate in every second) and then injected the traffic to network. According to injected traffic

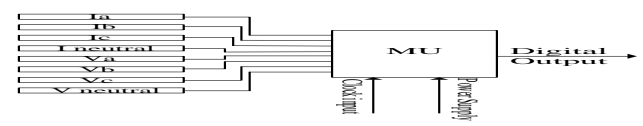

Figure 1. Merging Unit concept

Calculation of the behavior for the rest of the network component had been done [6], [7]. In [8] successful modeling for the MU, IED nodes and implementation of the process bus network based on the OPNET simulation tool, to estimate the SV traffic stream latency with different LAN topologies regarding to the IEC 61850-9-2LE. In this work illustration and discussion of the novel approach to estimate the sample value packets stream latency based on the processing time analysis for both source and destination. Moreover, the work shows the successful implementation of the novel SV packets stream latency estimation approach based on designing a practical IEC 61850-9-2LE process bus network using commercial physical devices that reflect a real behavior of the process bus network and to assess their performance operation within the SAS.

\section{NOVAL APPROACH TO ESTIMATE THE SV LATENCY}

Reliability and proper functionality of the MU plays major role for the implementation of the process bus network within the SAS. For instant incorrect measurements, delays and errors (bad quality SV data set) may lead to incorrect resolution of a protective and control IEDs that may cause in serious damages. Therefore within this part the SV traffic stream latency have been evaluated. The purpose of these evaluations and testing are to assess the performance operation, limits and capacity of the process bus network critical components such as
MU and the communication links are the focus of this study. The overall estimated SV traffic stream latency is composed of three different estimated latencies as illustrated in Fig. 2.

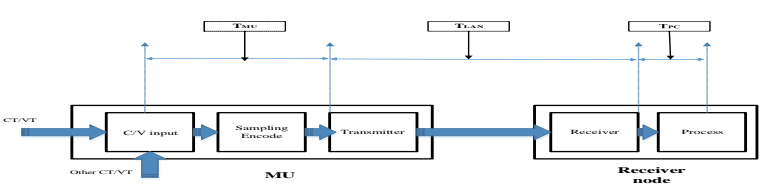

Figure 2. The SV packets latencies within the process bus network

Thus the estimated SV traffic stream latency (TESV) for each packet within the process bus network can be estimated as follows;

$\mathrm{TESV}=\mathrm{TMU}+\mathrm{TLAN}+\mathrm{TPC}$

Where, TMU: is the processing time for the MU to process (sampling, encapsulating) the data set and transmit the SV Ethernet packet to the destinations within the process bus network. TLAN: the estimated time required for the SV packets to travel from the source to their dissentions within the assigned process bus network. TPC: the estimated time required for the received node (PC in this work) to process and presents the received data. As a result the estimated SV latency (TELAN) for every packet within the assigned communication process bus network based on the novel latency estimation approach that the SV latency can be estimated from each two SV successive received packets as follows;

$$
\text { TELAN }=\text { TESV } 2-\text { TESV1 }
$$

Where, TESV2: is the second successive received SV packet, TESV1: is the first successive received SV packet. Since T2MU1 and T1MU1 are expected to be constant based on the processing time for the same length sequence of data and within the same device are expected to be constant. Also T2PC1 and T1PC1 are expected to be constant based on the processing time for packets with the same size and within the same device are expected to be constant reference [6] support this assumption. Nowhere, the estimated latency (TELAN) that has been experienced by each SV packet within the process bus communication network can be estimated as follows;

$$
\text { TELAN }=\text { T2LAN }- \text { T1LAN }
$$

As regard inter arrival time between the sequences of successive published SV packets (250 $\mu \mathrm{s})$ need to be considered within the calculation of the TELAN. From the discussion that had been mentioned above SV packets latency within the assigned process bus communication network has been successfully proved. Moreover implementation of the SV novel latency estimation approach has been made based on practical testing experiment based on two scenarios as follows in the next section. 


\section{CASE STUDY}

According to the first scenario design and implementation of the IEC 6180-9-2LE process bus network has been made within the DEMVE laboratory, the process bus network consists from OMICRON CMC 356, VAMP MU, CMC 850, CMIRIG-B, modern intelligent Ethernet switch, media convertors, cables and PC as illustrate in Fig 3. VAMP MU has been connected to the process bus network as a SV publisher. OMICRON CMC 356 and based on Quick CMC module within the Test Universe software has been used to inject the voltages and currents to the VAMP MU as illustrated in Fig 4. Whereas the PC has been configured to subscribe and analyze the receiving SV traffic stream packets using Wireshark to sniff the process bus network and define the SV packets receiving time. Whereas CMC 850 test set has been used as SV subscriber. Based on the subscription task CMC 850 has the ability to analyze the SV traffic, define the number of the received, lost SV packets, Moreover it has the ability to read the voltage and current values that associated with the SV packet frames based on metering and oscilloscope options as illustrated in Fig 5. It is worth noticing that all nodes are synchronous based on the assigned pulse per second (PPS) signal that has been generated from the CMC 356 as reference time based on its internal timebase.

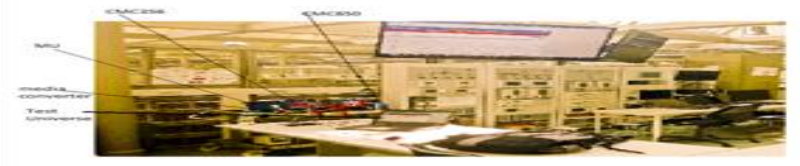

Figure 3. DEMVE laboratory

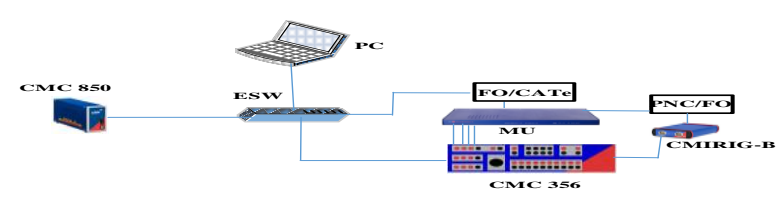

Figure 4. SV process bus design and connection digram

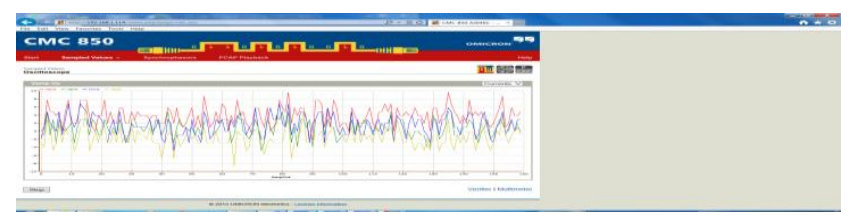

Figure 5. Current values within the VAMP MU SV

From Fig 4 the PPS signal has been generated from the CMC 356 and connected to the CMIRIG-B Interface box. CMIRIG-B enabling the connection between $\mathrm{CMC}$ test set and the devices that have the ability to send or receive the PPS or IRIG-B protocol signals. Moreover, it performs the level conversion between the CMC test set and the sources or receivers. The PPS signal output from the CMIRIG-
$\mathrm{B}$ need to be converted from $\mathrm{PNC} / \mathrm{FO}$ by using a media convector (E/O Convertor). Fiber optic medium has been used to connect between the CMIRIG-B PPS port and the MU PPS port. Nowhere the MU has been synchronized based on the CMC test set master clock. Within each PPS signal the MU sample counter start from 1 to 4000 that leads to synchronous different $\mathrm{SV}$ form different MU, also lets us know the order of sequential samples. Testing was performed in a live substation that $\mathrm{MU}$ provide the $\mathrm{SV}$ over the 1GBASE-FX that needs to convert to CAT5e by using a media convertor FO/CAT5e (BlackBox). CMC 356 test set has been used based on the QuickCMC test module to inject the analog three phase voltages and currents to the VAMP MU as illustrated in Fig 5. The SV output packet for the MU had been recorded for 4 seconds that results in 4 arrival measurements each related to the 1-PPS synchronizing signal. The VAMP MU publishes 4000 SV packets each second with a packet frame length 144 bytes. The inter-arrival between frames is $250 \mu \mathrm{s}$ resulting on 16 thousand SV packets measurements. SV packets arrival measurements have been recorded by using the Wireshark that has the ability to filter the network traffic. As regard SV traffic stream has been captured and exported to MATLAB. Within the MATLAB Environment cod had been written to analyze the recorded measurements, and from 3 calculations based on the novel approach to find the estimated latency (TELAN) that has been experienced by each SV packet within the process bus network has been made. Fig 6 illustrates the TELAN for the 16 thousand MU VAMP SV packet frames. Every packet had been experienced different latency based on the process bus communication network which is usual since every packet was send independently. These TELAN are within the acceptable range since the maximum latency when there is no packet loss is expected to be $250 \mu \mathrm{s}$. The mean and standard deviation of the TELAN for the captured frames was $($ mean $=2.50033 \mu \mathrm{s}),\left(\sigma=6.30073 * 10^{-6}\right)$. Moreover, in Fig 7 the TELAN for the captured frame were filtered based on the smpCnt $=0-3999$ and averaged to illustrates the behavior of the process bus network that the SV traffic stream latency has been shown every second.

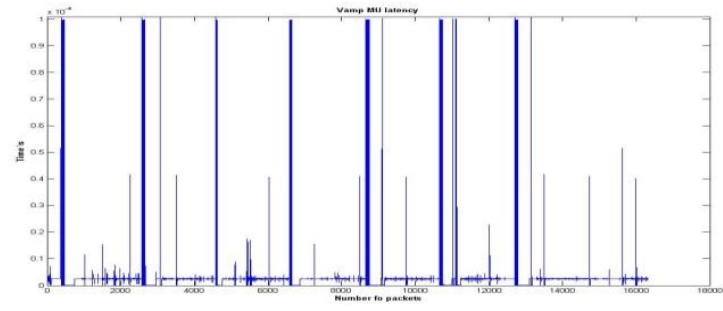

Figure 6. VAMP MU SV packets latency for 16000 packets 


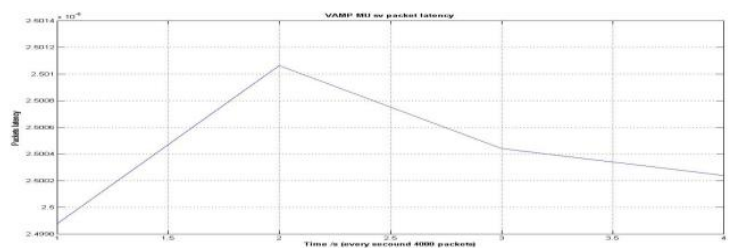

Figure 7. VAMP MU SV packets latency filtered and everged

Concerning to second scenario the OMICRON CMC 356 and based on SV module within the Test Universe software has been used as MU SV publisher as illustrate in Fig 8. QuickCMC module within the Test Universe software has been used to inject the voltages and current that the SV module need to published their values within the SV packet stream as illustrate in Fig 9. Whereas the PC has been configured to subscribe and analyze the receiving SV packets using Wireshark to sniff the process bus network and define the packets receiving time as illustrate in Fig 10. CMC 850 has been used as SV subscriber and analyzer as illustrate in Fig 11. All nodes have been synchronized as in scenario 1 . In case of using CMC test set as SV publisher no need for the media convertor since the CMC test set provide their SV packets over the RJ45 ETH1 or ETH2 ports.

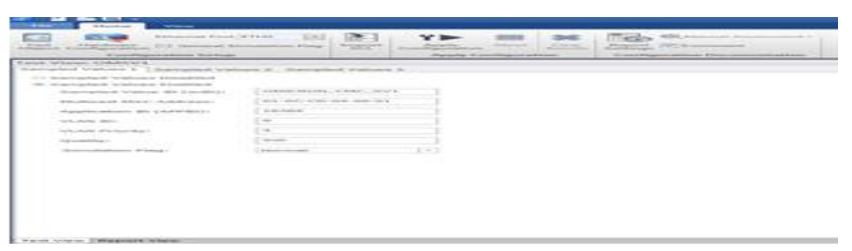

Figure 8. Test uneverse SV module

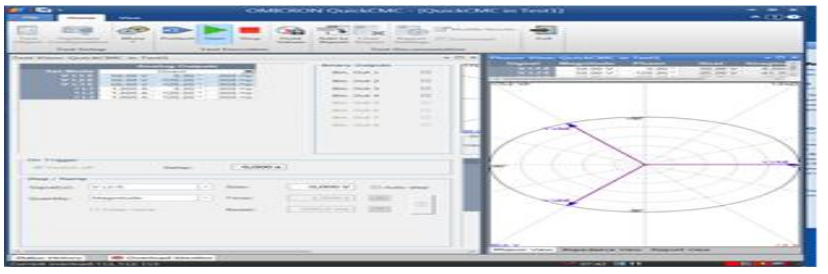

Figure 9. Test uneverse QuickCMC module

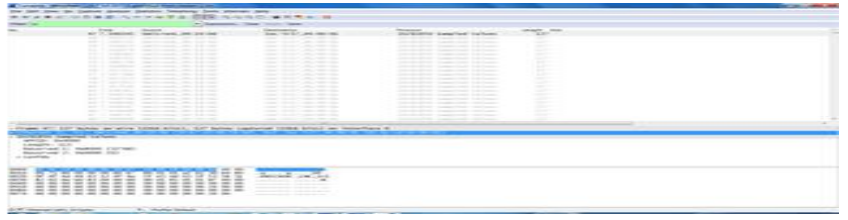

Figure 10. Wireshark network analyzer

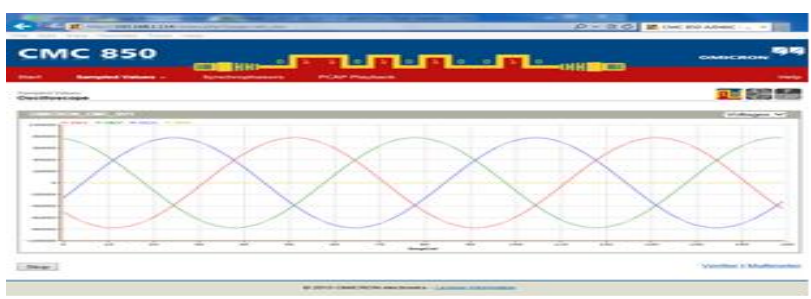

Figure 11. Test set CMC 850 subscriber and SV traffic analyzer
Testing was performed in a live substation that CMC 356 test set has been used to publish the SV traffic stream. The SV output packet had been recorded for 8 second that results in 8 arrival measurements each related to the 1-PPS synchronizing signal. The CMC 356 publishes 4000 SV packets every second with a packet frame length 127 bytes. The inter-arrival between frames is 250 $\mu \mathrm{s}$ resulting on 34 thousand SV packets measurements. SV packets arrival measurements have been recorded by using the Wireshark that has the ability to filter the network traffic. As regard SV traffic stream has been captured and exported to MATLAB. Within the MATLAB Environment analysis and calculation based on 3 for the recorded measurements to find the actual estimated latency (TELAN) that has been experienced by every SV packet within the process bus network has been made. Fig 12 illustrates the TELAN for the 34 thousand SV packet frames. Every packet had been experienced different latency based on the process bus communication network which is usual since every packet was send independently. These TELAN are within the acceptable range since the maximum latency when there is no packet loss is expected to be $250 \mu \mathrm{s}$.

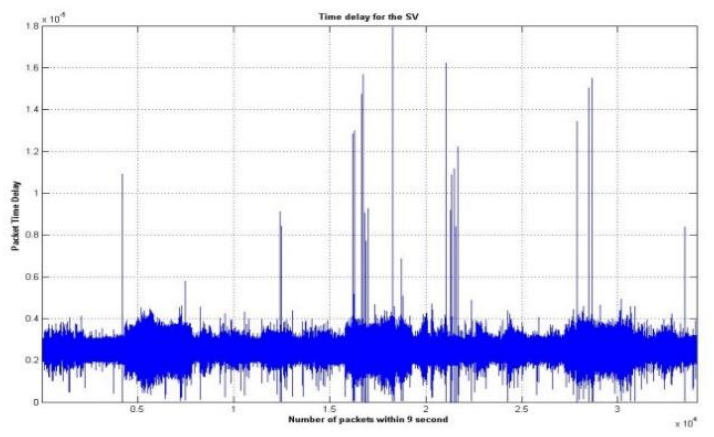

Figure 12. CMC $356 \mathrm{SV}$ packets latency for 35000 packets

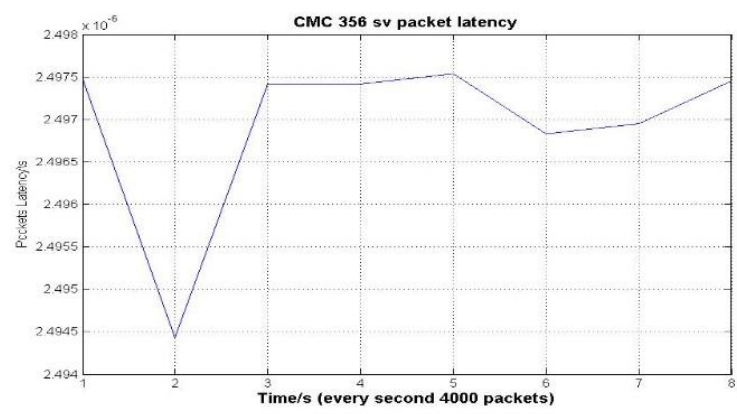

Figure 13. CMC $356 \mathrm{SV}$ packets filtered and everged

The mean and standard deviation of the $\mathrm{T}_{\text {elan }}$ for the captured frames was (mean $=2.49700 \mu \mathrm{s}),(\sigma=$ $\left.0.725500 * 10^{-6}\right)$. Moreover, in Fig 13 the Telan for the captured frame were filtered based on the smpCnt $=0-3999$ and averaged to illustrates the behavior of the process bus network that the SV traffic stream latency has been shown every second. The same idea that has been used in both scenarios 
can be used here were latencies have to be measured based on connecting several MUs within a process bus network to evaluate the limits and capacity of the process bus network critical components such the communication links and the Ethernet switch in which that may facilitate the design and guide the engineers to build the SAS in efficient way.

\section{CONCLUSION}

This paper illustrates and discusses the novel approach to estimate the sample value (SV) packets stream latency within LAN based on the IEC 618509-2LE by means of implementing of different practical laboratory testing within two scenarios. Testing is an important issue in IEC61850-9-2LE to highlight the capability for the process bus network and ensure the SV packets latency has been within the acceptable range before full scope integration of the SAS reaching "plug and play" solution. Within the laboratory testing in both scenarios advanced hardware and software tools had been demonstrated and used.

The obtaining results show that the successful implementation of the novel SV latency estimation approach based on designing a practical IEC 618509-2LE process bus using commercial physical devices. Furthermore, prove that the SV packet frames are compliant with the IEC 61850-9-2-LE criteria and the average of the SV packets latencies are within the acceptable range when no loss can be happen.

For future work, comparison the achieved results with the results that had been achieved from previous work in []. Furthermore, measuring the latencies for the SV traffic stream based on different bus topology that SV traffic stream needs to pass more than one Ethernet switch. Measuring the latencies for the SV traffic stream where more the one MU within the process bus network to define the limitation of the process bus network. Similar issues can be used to measure the accumulation jitter for the SV traffic stream when interfering with a traffic propagate in the same communication network in case of mixing the station level with the process level.

\section{REFERENCES}

[1] Communication Network and Sysstem for Power Utility Automation_part 9-2: Specific Communication Sevice Maping (SCSM)-Sampled Value Over ISO IEC8802-3, IEC 61850-9-2 ed2.0, 2011.

[2] "UCA International Users Group (2004, 7 Jul) Implementation Gudeline for Digital Interface to Instrument Transformer Using IEC 61850-9-2 R2-1," UCA Int Users Group, Raleigh, NC, USA, 2004.

[3] J. Amelot, Y.-S. Li-Baboud, C. Vasseur, J. Fletcher, D. Anand and J. Moyne "An IEEE 1588 performance testing dashboard for power industry requirements," in Proc, IEEE Int, Symp, Precis Clock Synchr meas Control Commun., Munich, Germany, Sep, 12-16, 2011.

[4] T. S. Sidhu, Y. Yin, K. "Modelling and Simulation for performance Evaluation of IEC61850-Based Substation Communicatiion System," IEEE Tran, Power Delivery, vol.22, no. 3, Jul. 2007. Jacobs and C. P. Bean, "Fine particles, thin films and exchange anisotropy," in Magnetism, vol. III, G. T. Rado and H. Suhl, Eds. New York, 1963.

[5] M. Gurbiel, P. Komarnick, Z. A. Styczynski, F. W. Gatzen, C. Dzienis, "Merging Unit Accuracy Testing," IEEE Power \& Energy General Meeting, Jul, 26-30, 2009.

[6] D. M. E. Ingram, R. R. Tylor, P. Schub, and D. A. Campbell, "Performance Analysis of IEC 61850 Sampled Value Process Bus Network," IEEE Transl. Industrial Information, vol. 9, no.3, Aug, 2013.

[7] D. M. E. Ingram, F. Steinhauser, C. Marinescu, R. R. Tylor, P. Schub, and D. A. Campbell, "Direct evaluation of IEC 61850-9-2 process bus network performance," IEEE Transl. Smart Grid, vol. 3, Dec, 2012.

[8] Mike Mekkanen, Reino Virrankoski, Mohammed Elmusrati \& Erkki Antila, "Using OPNET to Model and Evaluate the MU Performance Based on IEC61850-92LE," to appear in proc Conquering Complexity: Challenges and Opportunities conference November 3-5, 2014, Missouri University of Science \& Technology Philadelphia, PA, USA.

[9] Uzoamaka, L. Haiyn, C. Peter, A. Wen, Z. Ray and M Craig, "Performance Testing and Assessment of Merging Units using IEC 61850," in proc, Internation Conference on Advanced Power System Automation and Protection, Bejing, China,Oct, 2011 